\title{
The Approximated Solution for The Nonlinear Second Order Delay Multi-Value Problems
}

\author{
Eman. A. Abdul-Razzaq* \\ Received 18, December, 2008 \\ Accepted 16, December, 2009
}

\begin{abstract}
:
This paper is attempt to study the nonlinear second order delay multi-value problems. We want to say that the properties of such kind of problems are the same as the properties of those with out delay just more technically involved. Our results discuss several known properties, introduce some notations and definitions. We also give an approximate solution to the coined problems using the Galerkin's method.
\end{abstract}

Key words: Nonlinear Delay Multi-value Problems

\section{Introduction:}

Nonlinear second order delay multivalue problems are now recognized as an excellent source of models to simulate processes and phenomena observed in control theory, population dynamics, physics, chemistry, industrial robotics and many other fields, [1]. Due to this reason, they have been an object of active research. We shall note that, in spite of the great number of investigations of this kind of problems, their solutions has not yet elaborated like that of nonlinear second order multi-value problems, [2]. In recent years, there has been increasing interest on the solution of the nonlinear second order delay multi-value problems, [3].

In this work we use the Galerkin's method to solve the investigated problems.

\section{Basic Notations and Definitions}

In this section we introduce some notations and definitions that needed in this work. We start the section by the following definition.

\section{Definition 1.1}

The delay differential equation is an equation that the unknown function or it's derivative evaluated at arguments that differ by any fixed number of values, [1].

\section{Definition 1.2}

The delay differential equations are said to be nonlinear in case they are nonlinear with respect to the unknown function that enter with different arguments and their derivatives that appeared in them, [2].

The following definition introduce the new concepts of our results.

\section{Definition 1.3}

A nonlinear delay multi-value problem is a problem in which the unknown multi-function and some of its derivatives, evaluated at arguments which are different by any fixed number of values.

Consider the following nonlinear second order delay multi-value problem:

$$
\begin{aligned}
& -\left(p_{i}(x) y_{i}^{\prime}(x)\right)^{\prime}+q_{i}(x) y_{i}(x-\tau) \quad- \\
& f\left(x,\left\{\lambda_{i j}\right\}^{\mathrm{n}}{ }_{j=1}, y_{i}(x-\tau)\right)=0, \quad x \in[a, b] \ldots \\
& \text { [1.1] }
\end{aligned}
$$

with the boundary conditions:

*Department of Mathematics, College of Education- Ibn Al- Haitham, University of Baghdad 


$$
\begin{aligned}
& a_{i 1} y_{i}(a)=a_{i 2} y_{i}{ }^{\prime}(a) \\
& , x \in[a-\tau, a] \ldots[1.2] \\
& b_{i 1} y_{i}(b)=b_{i 2} y_{i}{ }^{\prime}(b) \\
& y_{i}(x-\tau)=\varphi_{i}(x-\tau) \text {, if } x-\tau<a
\end{aligned}
$$

where $p_{i}, p_{i}{ }^{\prime}$ and $q_{i}$ are given realvalued continuous functions defined on the interval $[a, b], p_{i}$ is positive, not both coefficients in one condition are zero, $\tau>0$ is the time delay, $f_{i}$ is a known nonlinear function with respect to $y_{i}, \varphi_{i}$ is the initial function defined on $x \in\left[x_{0}-\tau, x_{0}\right]$. The problem here is to determine the eigen-value $\lambda_{j}$ in which a nontrivial solution $y_{i}$ for the problem given by equations [1.1]-[1.2] occurs. In this case $\lambda_{j}$ is said to be a delay eigen-value and $y_{i}$ is the associated delay eigen-function.

More generally, if we rewrite the problem given by equations[1.1]-[1.2] as $\mathrm{L} y=0$ where

$$
L=\left[\begin{array}{cccc}
L_{1} & 0 & \ldots & 0 \\
0 & L_{2} & 0 & 0 \\
0 & \ldots & \ddots & 0 \\
0 & 0 & \ldots & L_{n}
\end{array}\right], \quad y=\left(\begin{array}{c}
y_{1} \\
y_{2} \\
\vdots \\
y_{n}
\end{array}\right)
$$

and

$L_{i}=-\frac{d}{d x^{2}} p_{i}(x)-\frac{d}{d x} p_{i}^{\prime}(x)+q_{i}(x) A_{i}(x), i=1,2, \ldots, n$ ... [1.3]

where $A_{i}(x)$ is an operator defined by $A_{i}(x) y_{i}(x)=y_{i}(x-\tau)$.

Thus, $L_{i}$ is a linear operator, which implies that $L$ is linear operator also, [3].

Like the nonlinear second order multi-value problems, the problem given by equations [1.1]-[1.2] satisfies the following notation, [4].

\section{Notation 1.1}

The linear operator given by equation [1.3] is self-adjoint, [5].

\section{Proposition 1.1}

The
$L=\left[\begin{array}{cccc}L_{1} & 0 & \ldots & 0 \\ 0 & L_{2} & 0 & 0 \\ 0 & \ldots & \ddots & 0 \\ 0 & 0 & \ldots & L_{n}\end{array}\right]$ is self-adjoint.

\section{Proof}

Directly from notation (1.1).

\section{Notation 1.2}

There are infinite number of eigenvalues forming a monotone increasing sequence with $\lambda_{i j} \rightarrow \infty$ as $j \rightarrow \infty$. Moreover, the eigen-functions corresponding to the eigen-values has exactly $j$ roots on the interval $(a, b)$ for each $i=1,2, \ldots, n,[6]$.

While the following remarks are also hold for the problem given by equations [1.1]-[1.2],[7].

\section{Remarks1.1}

1. All the delay eigen-values are real.

2. The delay eigen-functions are orthogonal.

3. The delay eigen-functions are complete and normal in $\mathrm{L}^{2}[a, b]$.

4. Each delay eigen-value corresponds only one delay eigenfunction in $\mathrm{L}^{2}[a, b]$.

To check the above remarks see [8].

\section{The Galerkin's Method}

In this section, we use the Galerkin's method to solve such a type of problems. To do this fix some $i$, say $i=k$, where $1 \leq k \leq n$. This method is based on approximating the unknown function $y_{k}$ as a linear combination of $n$ linearly independent functions $\left\{\phi_{i}\right\}^{n}{ }_{i=1}$, that is write

$$
y_{k}=\sum_{i=1}^{n} \phi_{i}(x) \ldots[2.1]
$$

which implies that

$$
y_{k}(x-\tau)=\sum_{i=1}^{n} \phi_{i}(x-\tau) \text {, if } x-\tau<a
$$

But, this approximated solution must satisfy the boundary conditions given by equations [1.2] to get a new 
approximated solution. By substituting this approximated solution into equation [1.1], one can get:

$$
\begin{aligned}
& R_{k}\left(x,\left\{\lambda_{i j}\right\}_{j=1}^{n}, \vec{c}\right)= \\
& -\left(p_{k}(x) \sum_{i=1}^{m} \phi_{i}^{\prime}(x)\right)^{\prime} \\
& +q_{k}(x) \sum_{i=1}^{m} \phi_{i}(x-\tau) \\
& -f_{k}\left(x,\left\{\lambda_{i j}\right\}_{j=1}^{n}, \sum_{i=1}^{m} \phi_{i}(x-\tau)\right) \ldots[2
\end{aligned}
$$

Where $R_{k}$ is the error in the approximation of equation [2.2] and $\vec{c}$ is the vector of $p$ elements of $c_{i}$, $i=1,2, \ldots, n$, where $m-2 \leq p \leq m$.

Next, choose $p+n$ linearly independent functions $\left\{\psi_{t}\right\}^{p+n}{ }_{t=1}$ to be orthogonal to $R_{k}$ on the interval $(a, b)$. That is,

$$
\int_{a}^{b} \psi_{i}(x) R_{k}\left(x,\left\{\lambda_{i j}\right\}_{j=1}^{n}, \vec{c}\right) d x=0 \quad, i=1,2, \ldots, n
$$

By evaluating the above equation at each $i=1,2, \ldots, n$ one can get a system of $p+n$ nonlinear equations with $p+n$ unknowns which can be solved by any suitable method to get the values of $\left\{\lambda_{i j}\right\}_{j=1}^{n}$ and $\vec{c}$, [9].

To illustrate the above method we shall give the following example.

\section{Example 2.1}

Consider the following nonlinear delay multi-value problem:

$$
\begin{gathered}
-y_{1}^{\prime \prime}(x)+(x-1)^{2} y_{1}(x-1)- \\
\left(2 \lambda_{1} y_{1}^{2}(x-1)-\lambda_{2}\right)=0 \ldots[2.3, \mathrm{a}] \quad, x \in[1,2] \\
-y_{2}^{\prime \prime}(x)+y_{2}(x-1)-\left(2 \lambda_{1} y_{2}(x-1)-\lambda_{2}\right)=0 \ldots[2.3, \mathrm{~b}] \\
\quad \text { with the boundary conditions: } \\
y_{1}(1)=0 \quad y_{1}(2)=\frac{1}{2} y_{1}^{\prime}(2) \ldots[2.4, \mathrm{a}] \\
y_{2}(1)=0 \quad 2 y_{2}(2)=y_{2}^{\prime}(2) \ldots[2.4, \mathrm{~b}] \\
y_{i}(x-1)=x-1, x \in[0,1]
\end{gathered}
$$

First, approximate the unknown function $y_{1}$ as a polynomial of degree three, which implies that $y_{1}(x-1)=$ $c_{1}+c_{2}(x-1)+c_{3}(x-1)^{2}+c_{4}(x-1)^{3}$ , but this approximated solution must satisfy the boundary conditions given by equations $[2.4, \mathrm{a}]$. Thus, $y_{1}(x-1)=$ $c_{3}(x-1)^{2}$, by substituting this approximated solution into equation $[2.3, \mathrm{a}]$ one can get:

$R_{1}\left(x, \lambda_{1}, \lambda_{2}, c_{3}\right)=-2 c_{3}+(x-1)^{2} c_{3}(x-$ $1)^{2}-\left(2 \lambda_{1} c_{3}(x-1)^{2}-\lambda_{2}\right) \ldots[2.5]$

Choose the functions $1, x, x^{2}$ and $x^{3}$ to be orthogonal to the error function $R_{1}$ to get the following system of equations:

$$
\begin{aligned}
& \int_{1}^{2} R_{1} d x=0 \\
& \int_{1}^{2} R_{1} x d x=0 \\
& \int_{1}^{2} R_{1} x^{2} d x=0 \\
& \int_{1}^{2} R_{1} x^{3} d x=0
\end{aligned}
$$

Thus, the nontrivial solution of the above system is $\lambda_{1}=\frac{1}{2}, \lambda_{2}=2$ and $c_{3}=1$.

Second, substitute the values of $\lambda_{1}$ and $\lambda_{2}$ into equation [2.3,b] to get that the solution of this equation is $y_{2}(x-1)$ $=d_{1}+d_{2}(x-1)+d_{3}(x-1)^{2}$.

But, this approximated solution must satisfy the boundary conditions given by equation [2.4,b]. Thus $d_{3}=1$.

$$
\text { Hence, }\left(\left(\frac{1}{2}, 2\right),\left((x-1)^{2},(x-1)^{2}\right)\right)
$$

is the double delay eigen-pair of the problem given by equations [2.3]-[2.4].

\section{Results:}

1. The nonlinear second order delay multi-value problems satisfies the 
same properties as those with out delay.

2. The Galerkin's method is a good choice to solve such a type of problems.

\section{Hint}

The above problems has been solved with the help of Math-Cad software package, and the programs are so easy that omitted.

\section{References:}

1. Peng, M., 2002, Oscillation Theorems of Second Order Nonlinear Neutral Delay Difference Equation, Comput. Math. Appl. 44:741-748.

2. Fu, X., Yan, B. and Liu, Y., 2005, Introduction of Impulsive Differential Systems, Science Press, Beijing.

3. Li, X., 2007, Oscillation Properties of Higher Order Impulsive Delay Difference Equation, Journal of Difference Equations. 2(2):209219.

4. Bainov, D., Lakshmikantham, V. and Simeonov, P., 1989, Theory of
Impulsive Differential Equations, World Scientific, Singapore.

5. Peng, M., 2000, Oscillation

Criteria for Second Order

Nonlinear Neutral Delay

Differential Equation, Comput.

Math. Appl. 39: 217-225.

6. Ballinger, G. and Liu, X., 2000, Existence, Uniqueness and Boundedness Results for Impulsive Daley Differential Equations, Applicable Analysis, 47: 71-93.

7. Xiaodi, L., 2008, Oscillation Properties of Second Order Differential-Difference Equation with Impulsive, ISSN 0973-5313, 3(1): 55-66.

8. Bhattacharyya, T., Binding, P. and Seddighi, K., 2001, Two Parameter Right Definite Sturm-Liouvill Problems with Eigenparameter Dependent Boundary Conditions, Proc, Roy. Soc. Edinburgh Sect., 131: 45-58.

9. Bainov, D., Dimitrova, M. and Dishlive, A., 2000, Oscillation of the Bounded Solutions of Impulsive Differential-Difference Equation of Second Order, Applied Mathematics and Computation. 114:61-68

\section{الحـل التقريبي للمسـائل اللاخطية التباطوئيه ازدواجية القيم الذاتية ذات الرتبة}

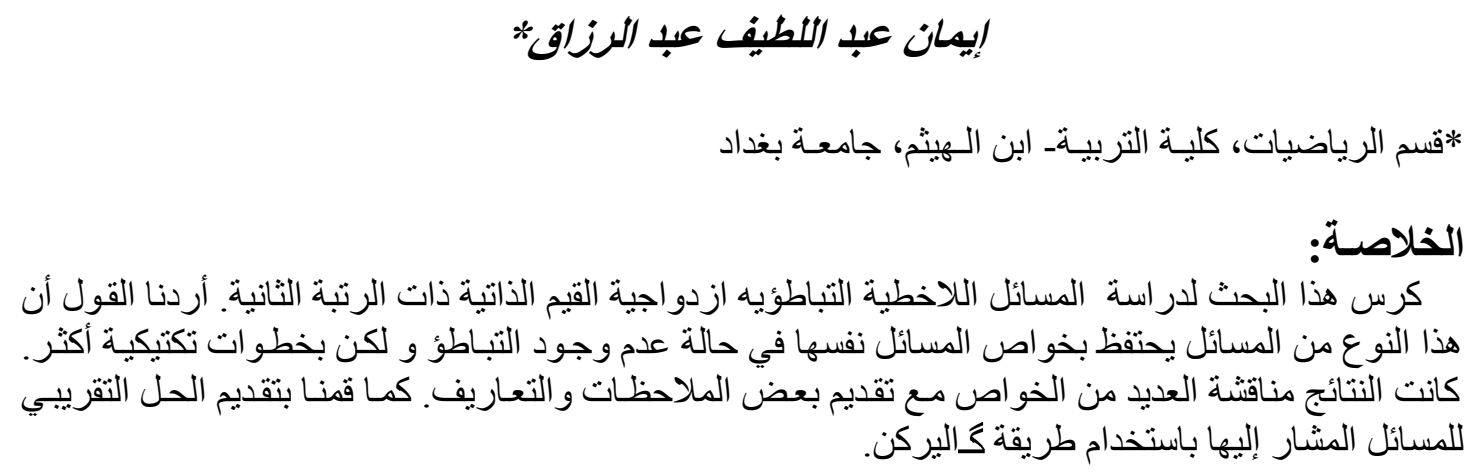

